\title{
The problem of the first return attached to a pseudodifferential operator in dimension 3
}

\author{
Oscar F. Casas-Sánchez ${ }^{a *}$, Jeanneth Galeano-Peñaloza $^{b}$, \\ JOHN J. RODRÍGUEZ-VEGA ${ }^{b}$ \\ ${ }^{a}$ Universidad de los Andes, Departamento de Matemáticas, Bogotá, Colombia. \\ ${ }^{b}$ Universidad Nacional de Colombia, Departamento de Matemáticas, Bogotá, Colombia.
}

\begin{abstract}
In this article we study the problem of first return associated to an elliptic pseudodifferential operator with non-radial symbol of dimension 3 over the $p$-adics.

Keywords: Random walks, ultradiffusion, $p$-adic numbers, non-archimedean analysis.

MSC2010: 82B41, 82C44, 26E30.

\section{El problema del primer retorno asociado a un operador seudodiferencial en dimensión 3}

Resumen. En este artículo estudiamos el problema del primer retorno asociado a un operador seudodiferencial elíptico con símbolo no radial de dimensión 3 sobre el cuerpo de los números $p$-ádicos.

Palabras clave: Caminatas aleatorias, ultradifusión, números p-ádicos, análisis no arquimediano.

\section{Introduction}

Avetisov et al. have constructed a wide variety of models of ultrametric diffusion constrained by hierarchical energy landscapes (see [2],[3]). From a mathematical point of view, in these models the time-evolution of a complex system is described by a $p$-adic master equation (a parabolic-type pseudodifferential equation) which controls the timeevolution of a transition function of a random walk on an ultrametric space, and the random walk describes the dynamics of the system in the space of configurational states which is approximated by an ultrametric space $\left(\mathbb{Q}_{p}\right)$.

\footnotetext{
*E-mail: oscasas@uniandes.edu.co

Received: 16 July 2015, Accepted: 20 August 2015.

To cite this article: O.F. Casas-Sánchez, J. Galeano-Peñaloza, J.J. Rodríguez-Vega, The problem of the first return attached to a pseudodifferential operator in dimension 3, Rev. Integr. Temas Mat. 33 (2015), No. 2, 107-119.
} 
The problem of the first return in dimension 1 was studied in [4], and in arbitrary dimension in [7]. In both articles, pseudodifferential operators with radial symbols were considered. More recently, Chacón-Cortés [6] considers pseudodifferential operators over $\mathbb{Q}_{p}^{4}$ with non-radial symbol; he studies the problem of first return for a random walk $X(t, w)$ whose density distribution satisfies certain diffusion equation.

In [5], the authors study elliptic pseudodifferential operators in dimension 3 and find a function, $Z(x, t), x \in \mathbb{Q}_{p}^{3}, t \in \mathbb{R}_{+}$, that satisfies the following equation

$$
\frac{\partial u(x, t)}{\partial t}=-\int_{\mathbb{Q}_{p}^{3}} K_{-\alpha}(y)(u(x-y, t)-u(x, t)) d^{3} y,
$$

where $K_{-\alpha}(x)$ is the Riesz kernel associated to the elliptic quadratic form $f^{\circ}(\xi)=p \xi_{1}^{2}+\xi_{2}^{2}-\epsilon \xi_{3}^{2}$.

Using the same techniques as in [6] we prove that the random walk $X(t, w)$ whose density distribution satisfies the equation (1) is recurrent if $\alpha \geq \frac{3}{2}$ and transient when $\alpha<\frac{3}{2}$. This result is analog to the one showed in [7], in the sense that $2 \alpha$ represent the degree of the symbol, and in this case the process is recurrent if $2 \alpha$ is greater that the dimension, 3 .

The article is organized as follows. In Section 2 we write some facts about $p$-adics. In Section 3 we define the symbol for the pseudodifferential operator and its Fourier transform. In Section 4 we study the Cauchy problem and give some properties of its fundamental solution, and define a Markov process over $\mathbb{Q}_{p}^{3}$. In Section 5 we determine the probability density function for a path of $X(t, \omega)$ goes back to $\mathbb{Z}_{p}^{3}$, and we show that the process is recurrent when $\alpha \geq \frac{3}{2}$, and otherwise is transient (see Theorem 5.7).

\section{Preliminars}

For the sake of completeness we include some preliminars. For more details the reader may consult [1],[9],[10].

\subsection{The field of $p$-adic numbers}

Along this article $p$ will denote a prime number different from 2. The field of $p$-adic numbers $\mathbb{Q}_{p}$ is defined as the completion of the field of rational numbers $\mathbb{Q}$ with respect to the $p$-adic norm $|\cdot|_{p}$, which is defined as

$$
|x|_{p}= \begin{cases}0 & \text { if } x=0, \\ p^{-\gamma} & \text { if } x=p^{\gamma} \frac{a}{b},\end{cases}
$$

where $a$ and $b$ are integers coprime with $p$. The integer $\gamma:=\operatorname{ord}(x)$, with ord $(0):=+\infty$, is called the $p$-adic order of $x$. We extend the $p$-adic norm to $\mathbb{Q}_{p}^{n}$ by taking

$$
\|x\|_{p}:=\max _{1 \leq i \leq n}\left|x_{i}\right|_{p}, \quad \text { for } x=\left(x_{1}, \ldots, x_{n}\right) \in \mathbb{Q}_{p}^{n} .
$$

We define $\operatorname{ord}(x)=\min _{1 \leq i \leq n}\left\{\operatorname{ord}\left(x_{i}\right)\right\}$; then $\|x\|_{p}=p^{-\operatorname{ord}(x)}$. Any $p$-adic number $x \neq 0$ has a unique expansion $x=p^{\operatorname{ord}(x)} \sum_{j=0}^{\infty} x_{j} p^{j}$, where $x_{j} \in\{0,1,2, \ldots, p-1\}$ and $x_{0} \neq 0$. 
By using this expansion, we define the fractional part of $x \in \mathbb{Q}_{p}$, denoted by $\{x\}_{p}$, as the rational number

$$
\{x\}_{p}= \begin{cases}0 & \text { if } x=0 \text { or } \operatorname{ord}(x) \geq 0, \\ p^{\operatorname{ord}(x)} \sum_{j=0}^{-\operatorname{ord}(x)-1} x_{j} p^{j} & \text { if } \operatorname{ord}(x)<0 .\end{cases}
$$

For $\gamma \in \mathbb{Z}$, denote by $B_{\gamma}^{n}(a)=\left\{x \in \mathbb{Q}_{p}^{n}:\|x-a\|_{p} \leq p^{\gamma}\right\}$ the ball of radius $p^{\gamma}$ with center at $a=\left(a_{1}, \ldots, a_{n}\right) \in \mathbb{Q}_{p}^{n}$, and take $B_{\gamma}^{n}(0):=B_{\gamma}^{n}$. Note that $B_{\gamma}^{n}(a)=B_{\gamma}\left(a_{1}\right) \times$ $\cdots \times B_{\gamma}\left(a_{n}\right)$, where $B_{\gamma}\left(a_{i}\right):=\left\{x \in \mathbb{Q}_{p}:\left|x_{i}-a_{i}\right|_{p} \leq p^{\gamma}\right\}$ is the one-dimensional ball of radius $p^{\gamma}$ with center at $a_{i} \in \mathbb{Q}_{p}$. The ball $B_{0}^{n}(0)$ is equal to the product of $n$ copies of $B_{0}(0):=\mathbb{Z}_{p}$, the ring of $p$-adic integers.

\subsection{The Bruhat-Schwartz space}

A complex-valued function $\varphi$ defined on $\mathbb{Q}_{p}^{n}$ is called locally constant if for any $x \in \mathbb{Q}_{p}^{n}$ there exists an integer $l(x) \in \mathbb{Z}$ such that

$$
\varphi\left(x+x^{\prime}\right)=\varphi(x) \text { for } x^{\prime} \in B_{l(x)}^{n} .
$$

A function $\varphi: \mathbb{Q}_{p}^{n} \rightarrow \mathbb{C}$ is called a Bruhat-Schwartz function (or a test function) if it is locally constant with compact support. The $\mathbb{C}$-vector space of Bruhat-Schwartz functions is denoted by $\mathbf{S}\left(\mathbb{Q}_{p}^{n}\right)$. For $\varphi \in \mathbf{S}\left(\mathbb{Q}_{p}^{n}\right)$, the largest of such numbers $l=l(\varphi)$ satisfying $(2)$ is called the exponent of local constancy of $\varphi$.

Let $\mathbf{S}^{\prime}\left(\mathbb{Q}_{p}^{n}\right)$ denote the set of all functionals (distributions) on $\mathbf{S}\left(\mathbb{Q}_{p}^{n}\right)$. All functionals on $\mathbf{S}\left(\mathbb{Q}_{p}^{n}\right)$ are continuous.

Set $\chi(y)=\exp \left(2 \pi i\{y\}_{p}\right)$ for $y \in \mathbb{Q}_{p}$. The map $\chi(\cdot)$ is an additive character on $\mathbb{Q}_{p}$, i.e. a continuos map from $\mathbb{Q}_{p}$ into $S$ (the unit circle) satisfying $\chi\left(y_{0}+y_{1}\right)=\chi\left(y_{0}\right) \chi\left(y_{1}\right)$, $y_{0}, y_{1} \in \mathbb{Q}_{p}$.

\subsection{Fourier transform}

Given $\xi=\left(\xi_{1}, \ldots, \xi_{n}\right)$ and $x=\left(x_{1}, \ldots, x_{n}\right) \in \mathbb{Q}_{p}^{n}$, we set $\xi \cdot x:=\sum_{j=1}^{n} \xi_{j} x_{j}$. The Fourier transform of $\varphi \in \mathbf{S}\left(\mathbb{Q}_{p}^{n}\right)$ is defined as

$$
(\mathcal{F} \varphi)(\xi)=\int_{\mathbb{Q}_{p}^{n}} \chi(\xi \cdot x) \varphi(x) d^{n} x \quad \text { for } \xi \in \mathbb{Q}_{p}^{n},
$$

where $d^{n} x$ is the Haar measure on $\mathbb{Q}_{p}^{n}$ normalized by the condition $\operatorname{vol}\left(B_{0}^{n}\right)=1$. The Fourier transform is a linear isomorphism from $\mathbf{S}\left(\mathbb{Q}_{p}^{n}\right)$ onto itself satisfying $(\mathcal{F}(\mathcal{F} \varphi))(\xi)=$ $\varphi(-\xi)$. We will also use the notation $\mathcal{F}_{x \rightarrow \xi} \varphi$ and $\hat{\varphi}$ for the Fourier transform of $\varphi$.

The Fourier transform $\mathcal{F}[f]$ of a distribution $f \in \mathbf{S}^{\prime}\left(\mathbb{Q}_{p}^{n}\right)$ is defined by

$$
(\mathcal{F}[f], \varphi)=(f, \mathcal{F}[\varphi]) \text { for all } \varphi \in \mathbf{S}\left(\mathbb{Q}_{p}^{n}\right) .
$$

The Fourier transform $f \rightarrow \mathcal{F}[f]$ is a linear isomorphism from $\mathbf{S}^{\prime}\left(\mathbb{Q}_{p}^{n}\right)$ onto $\mathbf{S}^{\prime}\left(\mathbb{Q}_{p}^{n}\right)$. Furthermore, $f=\mathcal{F}[\mathcal{F}[f](-\xi)]$. 


\subsection{The space $\mathfrak{M}_{\lambda}$}

We denote by $\mathfrak{M}_{\lambda}, \lambda \geq 0$, the $\mathbb{C}$-vector space of locally constant functions $\varphi(x)$ on $\mathbb{Q}_{p}^{n}$ such that $|\varphi(x)| \leq C\left(1+\|x\|_{p}^{\lambda}\right)$, where $C$ is a positive constant. If the function $\varphi$ depends also on a parameter $t$, we shall say that $\varphi \in \mathfrak{M}_{\lambda}$ uniformly with respect to $t$, if its constant $C$ and its exponent of local constancy do not depend on $t$.

\section{Pseudodifferential operators}

We take $f(\xi)=\epsilon \xi_{1}^{2}+p \epsilon \xi_{2}^{2}-p \xi_{3}^{2}$ and $f^{\circ}(\xi)=p \xi_{1}^{2}+\xi_{2}^{2}-\epsilon \xi_{3}^{2}$, with $\epsilon \in \mathbb{Z}$ a quadratic nonresidue module $p$. Given $\alpha>0$, we define the pseudodifferential operator with symbol $|f(\xi)|_{p}^{\alpha}$ by

$$
\begin{aligned}
\mathbf{S}\left(\mathbb{Q}_{p}^{3}\right) & \longrightarrow C\left(\mathbb{Q}_{p}^{3}\right) \cap L^{2}\left(\mathbb{Q}_{p}^{3}\right) \\
\varphi & \longrightarrow(\boldsymbol{f}(\partial, \alpha) \varphi)(x):=\mathcal{F}_{\xi \rightarrow x}^{-1}\left(|f(\xi)|_{p}^{\alpha} \mathcal{F}_{x \rightarrow \xi} \varphi\right) .
\end{aligned}
$$

In [5] the authors show that the Fourier transform of the symbol is given by

$$
\mathcal{F}\left[|f(x)|_{p}^{-\alpha}\right]=\frac{1-p^{-\alpha}}{1-p^{2 \alpha-3}}\left[\left(1+p^{\alpha-1}\right) I_{V_{1} \cup V_{\epsilon}}(x)+p^{\alpha-\frac{3}{2}}\left(p^{2-\alpha}+1\right) I_{V_{p}}(x)\right]\left|f^{\circ}(x)\right|^{\alpha-\frac{3}{2}},
$$

where

$$
I_{A}(x)= \begin{cases}1 & \text { if } x \in A, \\ 0 & \text { if } x \notin A,\end{cases}
$$

and for $\delta=1, \epsilon, p, p \epsilon$ put $V_{\delta}:=\left\{x \in \mathbb{Q}_{p}^{3} \mid f^{\circ}(x) \in \delta\left[\mathbb{Q}_{p}^{\times}\right]^{2}\right\}$. Observe that $V_{p \epsilon}=\emptyset$, otherwise the elliptic form $p x_{1}^{2}+x_{2}^{2}-\epsilon x_{3}^{2}-p \epsilon x_{4}^{2}=0$ would have non-trivial solution in $\mathbb{Q}_{p}^{4}$.

If we consider

$$
K_{\alpha}(x):=\frac{1-p^{-\alpha}}{1-p^{2 \alpha-3}}\left[\left(1+p^{\alpha-1}\right) I_{V_{1} \cup V_{\epsilon}}(x)+p^{\alpha-\frac{3}{2}}\left(p^{2-\alpha}+1\right) I_{V_{p}}(x)\right]\left|f^{\circ}(x)\right|^{\alpha-\frac{3}{2}},
$$

then equation (3) can be written as

$$
\hat{K}_{\alpha}(x)=|f(x)|^{-\alpha}, \quad \alpha \neq \frac{3}{2}+\frac{2 \pi \sqrt{-1}}{\ln p} \mathbb{Z},
$$

and as a distribution on $\sigma\left(\mathbb{Q}_{p}^{3}\right), K_{\alpha}(x)$ possesses a meromorphic continuation to all $\alpha \neq \frac{3}{2}+\frac{2 \pi \sqrt{-1}}{\ln p} \mathbb{Z}$ (see [5, Lemma 5]).

Since $\mathcal{F}^{-1}\left(|f|_{p}^{\alpha}\right)=K_{-\alpha}$ we have $|f(\xi)|_{p}^{\alpha} \mathcal{F}_{x \rightarrow \xi} \varphi \in L^{1}\left(\mathbb{Q}_{p}^{3}\right) \cap L^{2}\left(\mathbb{Q}_{p}^{3}\right)$, and the operator is well-defined. Therefore it is possible to write the operator as a convolution

$$
\boldsymbol{f}(\partial, \alpha) \varphi=K_{-\alpha} * \varphi=\int_{\mathbb{Q}_{p}^{3}} K_{-\alpha}(y)(\varphi(x-y)-\varphi(x)) d^{3} y,
$$


for $\varphi \in \mathbf{S}\left(\mathbb{Q}_{p}^{3}\right)$. Actually, the domain of the operator can be extended to the locally constant functions $u(x)$ such that

$$
\int_{\|x\|_{p} \geq 1} K_{-\alpha}(x)|u(x)| d^{3} x<\infty .
$$

There exists an important inequality for elliptic polynomials, which is essential to do all the calculations (see [11]). In our case, for $f$ and $f^{\circ}$, the inequalities are given in the next lemma.

Lemma 3.1 ([6, Lemma 3]). Let $f, f^{\circ}$ be as above. Then

(i) $p^{-1}\|x\|_{p}^{2} \leq|f(x)|_{p} \leq\|x\|_{p}^{2}$, for every $x \in \mathbb{Q}_{p}^{3}$,

(ii) $p^{-1}\|x\|_{p}^{2} \leq\left|f^{\circ}(x)\right|_{p} \leq\|x\|_{p}^{2}$, for every $x \in \mathbb{Q}_{p}^{3}$.

\section{The Cauchy problem}

The Cauchy problem

$$
\left\{\begin{array}{l}
\frac{\partial u(x, t)}{\partial t}=-\boldsymbol{f}(\partial, \alpha) u(x, t), \quad x \in \mathbb{Q}_{p}^{3}, \quad 0<t \leq T, \\
u(x, 0)=\varphi(x),
\end{array}\right.
$$

where $\alpha>0, T>0, \varphi \in \mathfrak{M}_{2 \lambda}, 0 \leq \lambda<\alpha$ has a solution $u: \mathbb{Q}_{p}^{3} \times[0, T] \rightarrow \mathbb{C}$ satisfying $u(x, t) \in \mathfrak{M}_{2 \lambda}$ and

$$
u(x, t):=Z(x, t) * \varphi(x)=\int_{\mathbb{Q}_{p}^{3}} Z(x-\eta, t) \varphi(\eta) d^{3} \eta,
$$

where the heat kernel $Z(x, t)$ attached to $f(x)$ is

$$
Z(x, t):=Z(x, t ; f, \alpha)=\int_{\mathbb{Q}_{p}^{3}} \chi(-\xi \cdot x) e^{-t|f(\xi)|_{p}^{\alpha}} d^{3} \xi,
$$

for $x \in \mathbb{Q}_{p}^{3}, t>0$ and $\alpha>0$ (see [5]).

Theorem 4.1. The function $Z(x, t)$ has the following properties:

(i) $Z(x, t) \geq 0$ for any $t>0$.

(ii) $\int_{\mathbb{Q}_{p}^{3}} Z(x, t) d^{3} x=1$ for any $t>0$.

(iii) $Z(x, t) \leq C t\left(\|x\|_{p}+t^{\frac{1}{2 \alpha}}\right)^{-3-2 \alpha}$, where $C$ is a positive constant, for any $t>0$ and any $x \in \mathbb{Q}_{p}^{3}$.

(iv) $Z(x, t) * Z\left(x, t^{\prime}\right)=Z\left(x, t+t^{\prime}\right)$ for any $t, t^{\prime}>0$.

Vol. 33, No. 2, 2015] 
(v) $\lim _{t \rightarrow 0^{+}} Z(x, t)=\delta(x)$ in $S^{\prime}\left(\mathbb{Q}_{p}^{3}\right)$.

(vi) $Z(x, t) \in C\left(\mathbb{Q}_{p}^{3}, \mathbb{R}\right) \cap L^{1}\left(\mathbb{Q}_{p}^{3}\right) \cap L^{2}\left(\mathbb{Q}_{p}^{3}\right)$ for any $t>0$.

Proof. See Theorems 1, 2, Proposition 2 and Corollary 1 of [11].

\section{1. $\quad$ Markov processes over $\mathbb{Q}_{p}^{3}$}

The space $\left(\mathbb{Q}_{p}^{3},\|\cdot\|_{p}\right)$ is a complete non-Archimedean metric space. Let $\mathcal{B}$ be the Borel $\sigma$-algebra of $\mathbb{Q}_{p}^{3}$; thus $\left(\mathbb{Q}_{p}^{3}, \mathcal{B}, d^{3} x\right)$ is a measure space. By using the terminology and results of $[8$, Chapters 2,3$]$, we set

$$
p(t, x, y):=Z(x-y, t) \text { for } t>0, x, y \in \mathbb{Q}_{p}^{3},
$$

and

$$
P(t, x, B)= \begin{cases}\int_{B} p(t, y, x) d^{3} y & \text { for } t>0, \quad x \in \mathbb{Q}_{p}^{3}, \quad B \in \mathcal{B}, \\ \mathbf{1}_{B}(x) & \text { for } t=0 .\end{cases}
$$

Lemma 4.2. With the above notation the following assertions hold:

(i) $p(t, x, y)$ is a normal transition density.

(ii) $P(t, x, B)$ is a normal transition function.

Proof. The result follows from Theorem 4.1 (see [8, Section 2.1] for further details).

Lemma 4.3. The transition function $P(t, x, B)$ satisfies the following two conditions:

$\mathbf{L}(\mathbf{B})$ For each $u \geq 0$ and compact $B$,

$$
\lim _{x \rightarrow \infty} \sup _{t \leq u} P(t, x, B)=0 .
$$

$\mathbf{M ( B )}$ For each $\epsilon>0$ and compact $B$,

$$
\lim _{t \rightarrow 0^{+}} \sup _{x \in B} P\left(t, x, \mathbb{Q}_{p}^{3} \backslash B_{\epsilon}^{3}(x)\right)=0 .
$$

Proof. (i) By Theorem 4.1 (iii) and the fact that $\|\cdot\|_{p}$ is an ultranorm, we have

$$
\begin{aligned}
P(t, x, B) & \leq C t \int_{B}\left(\|x-y\|_{p}+t^{\frac{1}{2 \alpha}}\right)^{-3-2 \alpha} d^{3} y \\
& =t\left(\|x\|_{p}+t^{\frac{1}{2 \alpha}}\right)^{-3-2 \alpha} \operatorname{vol}(B) \text { for } x \in \mathbb{Q}_{p}^{3} \backslash B .
\end{aligned}
$$

Therefore, $\lim _{x \rightarrow \infty} \sup _{t \leq u} P(t, x, B)=0$. 
(ii) By using Theorem 4.1 (iii), $\alpha>0$, and the fact that $\|\cdot\|_{p}$ is an ultranorm, we have

$$
\begin{aligned}
P\left(t, x, \mathbb{Q}_{p}^{3} \backslash B_{\epsilon}^{3}(x)\right) & \leq C t \int_{\|x-y\|_{p}>\epsilon}\left(\|x-y\|_{p}+t^{\frac{1}{2 \alpha}}\right)^{-3-2 \alpha} d^{3} y \\
& =C t \int_{\|z\|_{p}>\epsilon}\left(\|z\|_{p}+t^{\frac{1}{2 \alpha}}\right)^{-3-2 \alpha} d^{3} z \\
& \leq C t \int_{\|z\|_{p}>\epsilon}\|z\|_{p}^{-3-2 \alpha} d^{3} z \\
& =C^{\prime}(\alpha, \epsilon) t .
\end{aligned}
$$

Therefore,

$$
\lim _{t \rightarrow 0^{+}} \sup _{x \in B} P\left(t, x, \mathbb{Q}_{p}^{3} \backslash B_{\epsilon}^{3}(x)\right) \leq \lim _{t \rightarrow 0^{+}} \sup _{x \in B} C^{\prime}(\alpha, \epsilon) t=0 .
$$

Theorem 4.4. $Z(x, t)$ is the transition density of a time and space homogeneous Markov process which is bounded, right-continuous and has no discontinuities other than jumps.

Proof. The result follows from $\left[8\right.$, Theorem 3.6] by using that $\left(\mathbb{Q}_{p}^{3},\|x\|_{p}\right)$ is a semicompact space, i.e., a locally compact Hausdorff space with a countable base, and $P(t, x, B)$ is a normal transition function satisfying conditions $L(B)$ and $M(B)$ (cf. Lemmas 4.2 and 4.3).

\section{The first passage time}

The solution of the Cauchy problem

$$
\left\{\begin{array}{l}
\frac{\partial u(x, t)}{\partial t}=-\int_{\mathbb{Q}_{p}^{3}} K_{-\alpha}(y)[u(x-y)-u(x, t)] d^{3} y, \quad x \in \mathbb{Q}_{p}^{3}, \quad 0<t \leq T, \\
u(x, 0)=\Omega\left(\|x\|_{p}\right),
\end{array}\right.
$$

is given by

$$
u(x, t)=\int_{\mathbb{Q}_{p}^{3}} \chi(-\xi \cdot x) \Omega\left(\|\xi\|_{p}\right) e^{-t|f(\xi)|_{p}^{\alpha}} d^{3} \xi .
$$

Among other properties, the solution (10) is infinitely differentiable with respect to $t \geq 0$, and for $m \in \mathbb{N}$,

$$
\frac{\partial^{m} u}{\partial t^{m}}(x, t)=(-1)^{m} \int_{\mathbb{Q}_{p}^{3}}|f(\xi)|_{p}^{\alpha m} \chi(-\xi \cdot x) \Omega\left(\|\xi\|_{p}\right) e^{-t|f(\xi)|_{p}} d^{3} \xi .
$$

Vol. 33, No. 2, 2015] 
Lemma 5.1 ([5, Lemma 6]). For $\operatorname{Re}(\alpha)>0$ we have

$$
-\left(\int_{|x| \|_{p}>1} K_{-\alpha}(x) d^{3} x\right)=\frac{p^{-\alpha}\left(1-p^{-1}\right)\left(p^{\alpha}+p^{-1}+p^{-2}\right)}{1-p^{-2 \alpha-3}} \leq 1 .
$$

Definition 5.2. The random variable $\tau_{\mathbb{Z}_{p}^{3}}(\omega):=\tau(\omega): \mathbb{Q}_{p}^{3} \rightarrow \mathbb{R}^{+}$defined by

$$
\inf \left\{t>0 ; X(t, \omega) \in \mathbb{Z}_{p}^{3} \mid \text { there exists } t^{\prime} \text { such that } 0<t^{\prime}<t \text { and } X\left(t^{\prime}, \omega\right) \notin \mathbb{Z}_{p}^{3}\right\}
$$

is called the first passage time of a path of the random process $X(t, \omega)$ entering the domain $\mathbb{Z}_{p}^{3}$.

Note that the initial condition in (9) implies that

$$
\operatorname{Pr}\left(\left\{\omega \in \mathbb{Q}_{p}^{3} ; X(0, \omega) \in \mathbb{Z}_{p}^{3}\right\}\right)=1 .
$$

Definition 5.3. We say that $X(t, \omega)$ is recurrent with respect to $\mathbb{Z}_{p}^{3}$ if

$$
\operatorname{Pr}\left(\left\{\omega \in \mathbb{Q}_{p}^{3} ; \tau(\omega)<\infty\right\}\right)=1 .
$$

Otherwise we say that $X(t, \omega)$ is transient with respect to $\mathbb{Z}_{p}^{3}$.

The meaning of (12) is that every path of $X(t, \omega)$ is sure to return to $\mathbb{Z}_{p}^{3}$. If (12) does not hold, then there exist paths of $X(t, \omega)$ that abandon $\mathbb{Z}_{p}^{3}$ and never go back.

By using the same arguments given by Chacón in [6] we define the survival probability as

$$
S(t):=S_{\mathbb{Z}_{p}^{3}}(t)=\int_{\mathbb{Z}_{p}^{3}} \varphi(x, t) d^{3} x,
$$

which is the probability that a path of $X(t, \omega)$ remains in $\mathbb{Z}_{p}^{3}$ at the time $t$. Because there are no external forces acting on the random walk, we have

$S^{\prime}(t)=\left(\begin{array}{l}\text { Probability that a path of } X(t, \omega) \\ \text { goes back to } \mathbb{Z}_{p}^{3} \text { at the time } t\end{array}\right)-\left(\begin{array}{l}\text { Probability that a path of } X(t, \omega) \\ \text { exits } \mathbb{Z}_{p}^{3} \text { at the time } t\end{array}\right.$

$$
=g(t)-C \cdot S(t) \text { with } 0<C \leq 1 .
$$

In order to determine the probability density function $g(t)$ we compute $S^{\prime}(t)$. 


$$
\begin{aligned}
S^{\prime}(t) & =\int_{\mathbb{Z}_{p}^{3}} \frac{\partial \varphi(x, t)}{\partial t} d^{3} x=-\int_{\mathbb{Z}_{p}^{3}} \int_{\mathbb{Q}_{p}^{3}} K_{-\alpha}(y)[u(x-y, t)-u(x, t)] d^{3} y d^{3} x \\
& =-\int_{\mathbb{Z}_{p}^{3}} \int_{\|y\|_{p}>1} K_{-\alpha}(y)[u(x-y, t)-u(x, t)] d^{3} y d^{3} x \\
& =-\int_{\mathbb{Z}_{p}^{3}} \int_{\|y\|_{p}>1} K_{-\alpha}(y) u(x-y, t) d^{3} y d^{3} x+\int_{\mathbb{Z}_{p}^{3}} \int_{\|y\|_{p}>1} K_{-\alpha}(y) u(x, t) d^{3} y d^{3} x \\
& =-\int_{\|y\|_{p}>1} K_{-\alpha}(y) u(y, t) d^{3} y+\int_{\|y\|_{p}>1} K_{-\alpha}(y) d^{3} y \int_{\mathbb{Z}_{p}^{3}} u(x, t) d^{3} x \\
& =-\int_{\|y\|_{p}>1} K_{-\alpha}(y) u(y, t) d^{3} y-\left(\frac{p^{-\alpha}\left(1-p^{-1}\right)\left(p^{\alpha}+p^{-1}+p^{-2}\right)}{1-p^{-2 \alpha-3}}\right) S(t) .
\end{aligned}
$$

Therefore,

$$
g(t)=-\int_{\|y\|_{p}>1} K_{-\alpha}(y) u(y, t) d^{3} y
$$

and the constant $C:=\frac{p^{-\alpha}\left(1-p^{-1}\right)\left(p^{\alpha}+p^{-1}+p^{-2}\right)}{1-p^{-2 \alpha-3}}$ satisfies $0<C \leq 1$.

Proposition 5.4. The probability density function $f(t)$ of the random variable $\tau(\omega)$ satisfies the non-homogeneous Volterra equation of second kind

$$
g(t)=\int_{0}^{\infty} g(t-\tau) f(\tau) d \tau+f(t)
$$

Proof. The result follows from (14) by using the argument given in the proof of Theorem 1 in [4].

Lemma 5.5. For $f(x)=\epsilon x_{1}^{2}+p \epsilon x_{2}^{2}-p x_{3}^{2}$ and $\operatorname{Re}(s)>0$ the following formulas hold:

$$
\int_{\left.|| x\right|_{p}=1} \frac{1}{s+p^{-2 \gamma \alpha}|f(y)|_{p}^{\alpha}} d^{3} y=\frac{1-p^{-1}}{s+p^{-2 \gamma \alpha}}+\frac{p^{-1}\left(1-p^{-2}\right)}{s+p^{-2 \gamma \alpha-\alpha}} .
$$

(ii) If $\|\xi\|_{p} \geq p$, then there exist constants $C_{1}$ and $C_{2}$ such that

$$
\int_{\|x\|_{p}=1} \frac{\chi(y \cdot \xi)}{s+p^{-2 \gamma \alpha}|f(y)|_{p}^{\alpha}} d^{3} y= \begin{cases}\frac{C_{1}}{s+p^{-2 \gamma \alpha}}-\frac{C_{2}}{s+p^{-2 \gamma \alpha-\alpha}} & \text { if }\|\xi\|_{p}=p \\ 0 & \text { if }\|\xi\|_{p}>p\end{cases}
$$

Proof. By using the same technique as in [6, Lemma 15] we write $U:=\sqcup_{i} U^{(i)}$, where $U^{(i)}=U_{1}^{(i)} \times U_{2}^{(i)} \times U_{3}^{(i)}$ and

$$
U_{j}^{(i)}:= \begin{cases}p^{i_{j}} \mathbb{Z}_{p} & \text { if } i_{j}=1 \\ \mathbb{Z}_{p}^{*} & \text { if } i_{j}=0\end{cases}
$$

Vol. 33, No. 2, 2015] 
for $(i)=\left(i_{1}, i_{2}, i_{3}\right) \in\{0,1\}^{3} \backslash\{(1,1,1)\}$. Since $|f(y)|_{p} \in\left\{1, p^{-1}\right\}$, it is enough to compute the volume $\mu\left(U^{(i)}\right)$. The result follows from the following table.

\begin{tabular}{|c|c|c|}
\hline$(i)$ & $|f(y)|_{p}$ & $\mu\left(U^{(i)}\right)$ \\
\hline$(0,0,0)$ & 1 & $\left(1-p^{-1}\right)^{3}$ \\
\hline$(0,0,1)$ & 1 & $\left(1-p^{-1}\right)^{2} p^{-1}$ \\
\hline$(0,1,0)$ & 1 & $\left(1-p^{-1}\right)^{2} p^{-1}$ \\
\hline$(0,1,1)$ & 1 & $\left(1-p^{-1}\right) p^{-2}$ \\
\hline$(1,0,0)$ & $p^{-1}$ & $\left(1-p^{-1}\right)^{2} p^{-1}$ \\
\hline$(1,0,1)$ & $p^{-1}$ & $\left(1-p^{-1}\right) p^{-2}$ \\
\hline$(1,1,0)$ & $p^{-1}$ & $\left(1-p^{-1}\right) p^{-2}$ \\
\hline
\end{tabular}

Proposition 5.6. The Laplace transform $G(s)$ of $g(t)$ is given by $G(s)=G_{1}(s)+G_{2}(s)$, where

$$
\begin{aligned}
G_{1}(s)= & -\frac{p^{-\alpha}\left(1-p^{2 \alpha}\right)\left(1-p^{-1}\right)\left(p^{-1}+p^{-2}+p^{\alpha}\right)}{1-p^{-2 \alpha-3}} \\
& \times \sum_{\nu=1}^{\infty} p^{-2 \nu \alpha} \sum_{\gamma=\nu}^{\infty} p^{-3 \gamma}\left(\frac{1-p^{-1}}{s+p^{-2 \gamma \alpha}}+\frac{\left(1-p^{-2}\right) p^{-1}}{s+p^{-2 \gamma \alpha-\alpha}}\right),
\end{aligned}
$$

and

$$
\begin{aligned}
G_{2}(s)= & -\frac{p^{-\alpha}\left(1-p^{2 \alpha}\right)\left(1-p^{-1}\right)\left(p^{-1}+p^{-2}+p^{\alpha}\right)}{1-p^{-2 \alpha-3}} \\
& \times \sum_{\nu=1}^{\infty} p^{-2 \nu \alpha} p^{-3(\nu-1)}\left(\frac{C_{1}}{s+p^{-2(\nu-1) \alpha}}-\frac{C_{2}}{s+p^{-2(\nu-1) \alpha-\alpha}}\right) .
\end{aligned}
$$

Proof. We first note that, if $\operatorname{Re}(s)>0$, then

$$
K_{-\alpha}(x) e^{-s t} e^{-t|f(\xi)|_{p}^{\alpha}} \Omega\left(\|\xi\|_{p}\right) \in L^{1}\left((0, \infty) \times \mathbb{Q}_{p}^{3} \times \mathbb{Q}_{p}^{3} \backslash \mathbb{Z}_{p}^{3}, d t d^{3} \xi d^{3} x\right) .
$$

Therefore, by using (16) and Fubini's Theorem we have

$$
\begin{aligned}
G(s) & =\int_{0}^{\infty} e^{-s t} g(t) d t \\
& =-\int_{0}^{\infty} e^{-s t} \int_{\|x\|_{p}>1} K_{-\alpha}(x) u(x, t) d^{3} x d t \\
& =-\int_{0}^{\infty} e^{-s t} \int_{\|x\|_{p}>1} K_{-\alpha}(x) \int_{\mathbb{Q}_{p}^{3}} \chi(-\xi \cdot x) \Omega\left(\|\xi\|_{p}\right) e^{-t|f(\xi)|_{p}^{\alpha}} d^{3} \xi d^{3} x d t \\
& =-\int_{\|x\|_{p}>1} K_{-\alpha}(x) \int_{\mathbb{Z}_{p}^{3}} \chi(-\xi \cdot x) \int_{0}^{\infty} e^{-t\left(s+|f(\xi)|_{p}^{\alpha}\right)} d t d^{3} \xi d^{3} x \\
& =-\int_{\|x\|_{p}>1} K_{-\alpha}(x) \int_{\mathbb{Z}_{p}^{3}} \frac{\chi(-\xi \cdot x)}{s+|f(\xi)|_{p}^{\alpha}} d^{3} \xi d^{3} x .
\end{aligned}
$$


After the change of variables $x=p^{-\nu} y$ and $\xi=p^{\gamma} y^{\prime}$, and due to the fact that $K_{-\alpha}\left(p^{-\nu} y\right)=p^{-2 \nu \alpha-3 \nu} K_{-\alpha}(y)$, we obtain

$$
G(s)=-\sum_{\nu=1}^{\infty} p^{-2 \nu \alpha} \int_{\|y\|_{p}=1} K_{-\alpha}(y) \sum_{\gamma=0}^{\infty} p^{-3 \gamma} \int_{\left\|y^{\prime}\right\|_{p}=1} \frac{\chi\left(-p^{-\nu+\gamma} y \cdot y^{\prime}\right)}{s+p^{-2 \alpha \gamma}\left|f\left(y^{\prime}\right)\right|_{p}^{\alpha}} d^{3} y^{\prime} d^{3} y
$$

In order to calculate the interior integral we split the set $\left\|y^{\prime}\right\|_{p}=1$ into two parts, when $\left\|p^{-\nu+\gamma} y \cdot y^{\prime}\right\|_{p} \leq 1$, and when $\left\|p^{-\nu+\gamma} y \cdot y^{\prime}\right\|_{p}>1$. The first case occurs when $\gamma \geq \nu$, and then $\chi\left(-p^{-\nu+\gamma} y \cdot y^{\prime}\right)=1$. The second case occurs when $\gamma=0, \ldots, \nu-1$. By Lemma 5.5 $G(s)$ takes the form $G(s)=G_{1}(s)+G_{2}(s)$, where

$$
\begin{aligned}
G_{1}(s):= & -\sum_{\nu=1}^{\infty} p^{-2 \nu \alpha} \int_{\|y\|_{p}=1} K_{-\alpha}(y) \sum_{\gamma=\nu}^{\infty} p^{-3 \gamma} \int_{\left\|y^{\prime}\right\|_{p}=1} \frac{1}{s+p^{-2 \alpha \gamma}\left|f\left(y^{\prime}\right)\right|_{p}^{\alpha}} d^{3} y^{\prime} d^{3} y \\
= & -\frac{p^{-\alpha}\left(1-p^{2 \alpha}\right)\left(1-p^{-1}\right)\left(p^{-1}+p^{-2}+p^{\alpha}\right)}{1-p^{-2 \alpha-3}} \\
& \times \sum_{\nu=1}^{\infty} p^{-2 \nu \alpha} \sum_{\gamma=\nu}^{\infty} p^{-3 \gamma}\left(\frac{1-p^{-1}}{s+p^{-2 \gamma \alpha}}+\frac{\left(1-p^{-2}\right) p^{-1}}{s+p^{-2 \gamma \alpha-\alpha}}\right)
\end{aligned}
$$

and

$$
\begin{aligned}
G_{2}(s):= & -\sum_{\nu=1}^{\infty} p^{-2 \nu \alpha} \int_{\|y\|_{p}=1} K_{-\alpha}(y) \sum_{\gamma=0}^{\nu-1} p^{-3 \gamma} \int_{\left\|y^{\prime}\right\|_{p}=1} \frac{\chi\left(-p^{-\nu+\gamma} y \cdot y^{\prime}\right)}{s+p^{-2 \alpha \gamma}\left|f\left(y^{\prime}\right)\right|_{p}^{\alpha}} d^{3} y^{\prime} d^{3} y \\
= & -\frac{p^{-\alpha}\left(1-p^{2 \alpha}\right)\left(1-p^{-1}\right)\left(p^{-1}+p^{-2}+p^{\alpha}\right)}{1-p^{-2 \alpha-3}} \\
& \times \sum_{\nu=1}^{\infty} p^{-2 \nu \alpha} p^{-3(\nu-1)}\left(\frac{C_{1}}{s+p^{-2(\nu-1) \alpha}}-\frac{C_{2}}{s+p^{-2(\nu-1) \alpha-\alpha}}\right) .
\end{aligned}
$$

Theorem 5.7. (i) If $\alpha \geq \frac{3}{2}$, then $X(t, \omega ; \boldsymbol{W})$ is recurrent with respect to $\mathbb{Z}_{p}^{3}$.

(ii) If $\alpha<\frac{3}{2}$, then $X(t, \omega ; \boldsymbol{W})$ is transient with respect to $\mathbb{Z}_{p}^{3}$.

Proof. By Proposition 5.4, the Laplace transform $F(s)$ of $f(t)$ equals $\frac{G(s)}{1+G(s)}$, where $G(s)$ is the Laplace transform of $g(t)$, and thus

$$
F(0)=\int_{0}^{\infty} f(t) d t=1-\frac{1}{1+G(0)}
$$

Hence, in order to prove that $X(t, \omega ; \boldsymbol{W})$ is recurrent is sufficient to show that $G(0)=$ $\lim _{s \rightarrow 0} G(s)=\infty$, and to prove that it is transient, that $G(0)=\lim _{s \rightarrow 0} G(s)<\infty$.

(i) Take $s \in \mathbb{R}, s>0$ and set $s=p^{-2 \nu \alpha}=p^{-2 \gamma \alpha}$; note that $s \rightarrow 0^{+} \Leftrightarrow v \rightarrow \infty(v=\gamma)$. Now, taking only the first term of $G_{1}(s)$ we have

Vol. 33, No. 2, 2015] 


$$
\begin{aligned}
G(s)> & -\frac{p^{-\alpha}\left(1-p^{2 \alpha}\right)\left(1-p^{-1}\right)\left(p^{-1}+p^{-2}+p^{\alpha}\right)}{1-p^{-2 \alpha-3}} \\
& \times \sum_{\gamma=1}^{\infty} p^{-3 \gamma}\left(\frac{1-p^{-1}}{s+p^{-2 \gamma \alpha}}+\frac{\left(1-p^{-2}\right) p^{-1}}{s+p^{-2 \gamma \alpha-\alpha}}\right)+G_{2}(s) .
\end{aligned}
$$

We get $G_{2}\left(p^{-2 \nu \alpha}\right)<\infty$, but the first sum diverges if $\alpha \geq \frac{3}{2}$. Then,

$$
\lim _{s \rightarrow 0^{+}} G(s)=\infty .
$$

(ii) Now

$$
\begin{aligned}
|G(s)| \leq & -\frac{p^{-\alpha}\left(1-p^{2 \alpha}\right)\left(1-p^{-1}\right)\left(p^{-1}+p^{-2}+p^{\alpha}\right)}{1-p^{-2 \alpha-3}} \\
& \times \sum_{\nu=1}^{\infty} p^{-2 \nu \alpha} \sum_{\gamma=\nu}^{\infty} p^{-3 \gamma}\left(\frac{1-p^{-1}}{p^{-2 \gamma \alpha}}+\frac{\left(1-p^{-2}\right) p^{-1}}{p^{-2 \gamma \alpha-\alpha}}\right)+G_{2}(0) .
\end{aligned}
$$

One sees easily that $G_{2}(0)$ converges, and that the double series converges if $\alpha>\frac{3}{2}$. Therefore $\lim _{s \rightarrow 0^{+}} G(s)<\infty$.

\section{References}

[1] Albeverio S., Khrennikov A.Y. and Shelkovich V.M., Theory of p-adic distributions: linear and nonlinear models, London Mathematical Society Lecture Note Series, 370, Cambridge University Press, Cambridge, 2010.

[2] Avetisov V.A. and Bikulov A.K., "On the ultrametricity of the fluctuation dynamic mobility of protein molecules" (Russian) Tr. Mat. Inst. Steklova 265 (2009), Izbrannye Voprosy Matematicheskaya Fiziki i p-adicheskaya Analiza, 82-89; translation in Proc. Steklov Inst. Math. 265 (2009), No. 1, 75-81.

[3] Avetisov V.A., Bikulov A.K. and Kozyrev S.V., "Description of logarithmic relaxation by a model of a hierarchical random walk", (Russian) Dokl. Akad. Nauk 368 (1999), No. 2, $164-167$.

[4] Avetisov V.A., Bikulov A.K. and Zubarev A.P., "First passage time distribution and the number of returns for ultrametric random walks", J. Phys. A 42 (2009), No. 8, 18 pp.

[5] Casas-Sánchez O.F., Galeano-Peñaloza J. and Rodríguez-Vega J.J., "Parabolic-type pseudodifferential equations with elliptic symbols in dimension 3 over $p$-adics", $p$-Adic Numbers Ultrametric Anal. Appl. 7 (2015), No. 1, 1-16.

[6] Chacón-Cortés L.F., "The problem of the first passage time for some elliptic pseudodifferential operators over the p-adics", Rev. Colombiana Mat. 48 (2014), No. 2, 191-209.

[7] Chacón-Cortés L.F. and Zúñiga-Galindo W.A., "Nonlocal operators, parabolic-type equations, and ultrametric random walks", J. Math. Phys. 54 (2013), No. 11, 17 pp.

[Revista Integración 
[8] Dynkin E.B., Markov processes Vol. I. Die Grundlehren der Mathematischen Wissenschaften, Bände 121, Academic Press Inc., Publishers, New York; Springer-Verlag, Berlin-Göttingen-Heidelberg 1965.

[9] Kochubei A.N., Pseudo-differential equations and stochastics over non-Archimedian fields. Monographs and Textbooks in Pure and Applied Mathematics, 244. Marcel Dekker, Inc., New York, 2001.

[10] Vladimirov V.S., Volovich I.V. and Zelenov E.I., p-adic analysis and mathematical physics. Series on Soviet and East European Mathematics, 1. World Scientific Publishing Co., Inc., River Edge, NJ, 1994.

[11] Zúñiga-Galindo W.A., "Parabolic equations and Markov processes over $p$-adic fields", Potential Anal. 28 (2008), No. 2, 185-200. 